Relations industrielles

Industrial Relations

\title{
Wemer Sengenberger, Gary W. Loveman and Michael J. Piore. The Re-Emergence of Small Enterprises. Industrial Restructuring in Industrialised Countries
}

\section{Ginette Dussault}

Volume 47, numéro 3, 1992

URI : https://id.erudit.org/iderudit/050803ar

DOI : https://doi.org/10.7202/050803ar

Aller au sommaire du numéro

Éditeur(s)

Département des relations industrielles de l'Université Laval

ISSN

0034-379X (imprimé)

1703-8138 (numérique)

Découvrir la revue

Citer ce compte rendu

Dussault, G. (1992). Compte rendu de [Wemer Sengenberger, Gary W. Loveman and Michael J. Piore. The Re-Emergence of Small Enterprises. Industrial

Restructuring in Industrialised Countries]. Relations industrielles / Industrial Relations, 47(3), 587-590. https://doi.org/10.7202/050803ar

Tous droits réservés (c) Département des relations industrielles de l'Université Laval, 1992
Ce document est protégé par la loi sur le droit d'auteur. L'utilisation des services d'Érudit (y compris la reproduction) est assujettie à sa politique d'utilisation que vous pouvez consulter en ligne.

https://apropos.erudit.org/fr/usagers/politique-dutilisation/ 
ces termes3. Un autre exemple est la manie d'utiliser «pour un dollar et autres considérations» qui est inspiré directement de la common law selon laquelle la considération (le fameux pepper grain du traité de droit contractuel anglais) fonde l'existence du contrat, par opposition à la rencontre des volontés qui préside à la formation des contrats en droit civil.

La partie 2 est, à mon avis, mieux réussie que la première non pas qu'elle soit plus originale mais en ce qu'elle ne fait pas (ou fait moins) double emploi avec la doctrine récente publiée sur le contrat individuel de travail en Québec.

Ce volume sera utile aux praticiens, et je dirais même aux étudiants universitaires qui suivraient un cours élaboré sur le contrat individuel de travail. Ils auraient beaucoup à y apprendre sur le thème de la rédaction des actes juridiques.

Ce livre d'accès facile devrait faire partie de la bibliothèque des spécialistes et des praticiens du droit du travail. En même temps, on ne peut s'empêcher de noter qu'un véritable traité sur les rapports individuels du travail reste à écrire. Avis aux générations montantes.

Claude D'Aoust

Université de Montréal

The Re-Emergence of Small Enterprises. Industrial Restructuring in Industrialised Countries, edited by Werner Sengenberger, Gary W. Loveman and Michael J. Piore, Genève, International Institute for Labour Studies, 1990, 308 p., ISBN 92-9014-465-3

L'Institut international d'études sociales (BIT) publie un recueil de six monographies nationales ainsi qu'une synthèse de ces différentes études portant sur la réalité des petites et moyennes entreprises dans les pays industrialisés. Chaque monographie est rédigée par un chercheur du pays en question. Les pays retenus et les auteurs des monographies sont les suivants: la France, avec Jean-François Amadieu; la République fédérale d'Allemagne, avec Stephanie Weimer; I'Italie, avec Giacomo Becattini; le Japon, avec Kazutoshi Koshiro; le Royaume-Uni, avec David Marsden et finalement les ÉtatsUnis, avec Michael J. Piore. Le chapitre d'introduction, qui constitue en fait la synthèse des différentes monographies nationales, est rédigé par Gary Loveman et Werner Sengenberger.

L'intérêt de l'IIES pour cette question s'explique par le fait que la part de l'emploi dans les PME a augmenté partout depuis la fin des années 70 . Étant donné que les conditions de travail varient selon la taille des entreprises, il était normal que l'organisme chargé de l'établissement des normes internationales de travail se préoccupe de la question. Chaque monographie nationale essaie donc de répondre à une même série de questions, à savoir l'importance quantitative du

3 Voir le Dictionnaire de droit privé et Lexiques bilingues, Centre de recherche de droit privé \& comparé du Québec, deuxième édition, Cowansville (Québec), Les Éditions Yvon Blais, 1991, p. 120. 
phénomène de la résurgence des PME; les explications généralement avancées à l'intérieur de chacun des pays; les conditions de travail dans ces PME; la place et le rôle des PME dans la structure industrielle nationale, en particulier leur degré de dépendance face aux grandes entreprises, et les modifications, s'il y a lieu, du contexte législatif ou réglementaire ayant favorisé le développement récent des PME. En toile de fond de ces différentes monographies, il y a aussi la préoccupation de vérifier si l'analyse de Piore et Sabel prévoyant une modification profonde des structures productives (thèse de la spécialisation flexible développée dans The Second Industrial Divide) en faveur d'unités de production plus petites et plus flexibles peut expliquer le phénomène.

Le chapitre d'introduction est, en fait, davantage que la synthèse des monographies nationales. II prend même certaines libertés par rapport aux conclusions de ces dernières dont on ne se rend compte qu'en lisant chacune d'elles. Dans un premier temps, la problématique est exposée. Suit une discussion assez aride des problèmes méthodologiques entourant l'interprétation des données sur l'évolution de la part de l'emploi total attribuée aux PME. Sont ensuite analysés les écarts de salaire entre PME et grandes entreprises ainsi que les régimes de négociation collective dans les différents pays. Ces premières sections sont particulièrement intéressantes pour la mise en lumière des différences internationales dans les structures industrielles: la part de l'emploi dans les entreprises de 500 employés et plus varie en effet de $18 \%$ en Italie à plus de $40 \%$ aux États-Unis et en Allemagne, le Japon occupant une position intermédiaire avec seulement $27 \%$ de sa main-d'oeuvre dans les grandes entreprises. Quant aux écarts de salaire, on constate qu'ils sont beaucoup plus grands aux États-Unis qu'en Europe ou au Japon: les PME américaines versent des salaires équivalant à $57 \%$ des salaires des grandes entreprises alors que les salaires des PME allemandes atteignent $90 \%$ du salaire des grandes entreprises. Que des structures industrielles si différentes au départ aient connu une mème évolution au cours des années 80 , soit un renversement de la tendance historique vers la concentration de la maind'oeuvre dans les entreprises de grande taille, mérite donc une analyse attentive.

Cette tendance historique se vérifiait pour tous les pays disposant de données chronologiques et ne manifestait qu'un seul épisode anormal, soit la grande dépression des années 30, période pendant laquelle la part de l'emploi dans les PME avait augmenté. Cette expérience historique avait donc implicitement établi que les grandes unités de production bénéficiaient d'avantages comparatifs absolus et donc que le progrès économique passait nécessairement par une plus grande concentration de l'activité de production. Que cette tendance ait été inversée dans les années 80 et, plus fondamentalement peut-être, que le discours économique officiel laisse entendre que le succès de la reprise économique et que la croissance future dépendent dorénavant de la performance des petites et moyennes entreprises bouleversent donc la 'sagesse' traditionnelle. $Y$ a-t-il vraiment des changements fondamentaux à l'oeuvre qui modifient les avantages comparés des grandes et des petites entreprises ou n'assiste-t-on qu'à la répétition du phénomène déjà observé dans les années 30 (déviation temporaire de la tendance à long terme, provoquée par la profondeur de la crise économique)? 
C'est dans la réponse apportée à cette question que le chapitre d'introduction prend le plus de liberté face aux monographies nationales. En effet, le chapitre d'introduction fait le tour des explications le plus souvent avancées et conclut que celle qui semble s'appliquer le mieux à l'ensemble des diverses expériences nationales est celle de la spécialisation flexible. Les autres explications envisagées sont les suivantes: l'illusion statistique (reflétant le développement du tertiaire et l'éloignement temporaire de la taille optimale des entreprises); l'effet conjoncturel (semblable à ce qui a été observé durant les années 30); l'avantage coût des PME (conséquence de l'accroissement des écarts de salaire entre PME et grandes entreprises); la libéralisation et la dérèglementation du marché du travail (d'une part, l'allégement des réglementations du marché du travail contribue à l'essor du dynamisme inhérent aux PME et d'autre part, les employeurs utilisent les PME pour affaiblir le mouvement syndical afin de regagner et maintenir une flexibilité du marché du travail).

Analysées indépendamment les unes des autres, les expériences nationales pointeraient dans des directions différentes en ce qui a trait à l'explication retenue. Par exemple, la monographie allemande insiste sur l'explication structurelle (importance grandissante du tertiaire) et sur la conjoncture économique générale (taux de chómage élevé) pour expliquer le développement récent de l'emploi dans les PME. Pour l'auteure de cette monographie, les tendances à la décentralisation qui émaneraient des grandes entreprises elles-mêmes (thèse de Piore et Sabel) ne sont pas particulièrement marquées dans ce pays. La monographie japonaise fournit des données qualitatives plus directement utiles au soutien de la thèse de la spécialisation flexible (diminution de l'écart technologique entre grandes et petites entreprises, redéfinition des liens entre donneurs d'ordre et sous-traitants en faveur d'une reconnaissance de leur interdépendance mutuelle plutôt que de la subordination des petites entreprises) mais apporte aussi des données quantitatives plus nuancées: la part de l'emploi total fourni par les PME aurait recommancé à diminuer au début de la décennie 80 , ce qui ne nous permet pas d'écarter facilement l'hypothèse que la remontée précédente n'était qu'un incident temporaire, semblable à celui observé au cours des années 30 . Même la monographie américaine, rédigée par Piore lui-même, conclut que les données qualitatives ne permettent pas d'arriver à une réponse claire quant aux causes du développement récent des PME. II y aurait bien deux raisons, distinctes l'une de l'autre: profiter des plus bas coûts de main-d'oeuvre (les écarts de rémunération ont augmenté au cours des 15 dernières années) et implanter de nouvelles techniques de production plus flexibles. Si les auteurs du chapitre synthèse donnent tout de même la première place à l'explication de la spécialisation flexible, c'est qu'ils admettent difficilement qu'une évolution identique survenue dans des économies très différentes au départ quant à leur structure industrielle ne partage pas une cause commune mais relèverait d'une logique différente d'un pays à l'autre. Or, si explication commune il doit y avoir, celle de la spécialisation flexible doit certainement recevoir une attention particulière.

La dernière partie du chapitre d'introduction traite du contexte institutionnel nécessaire au développement de PME productives. Cette partie se veut plus prospective. Étant donné que l'observation de la réalité nous enseigne 
que les petites entreprises ont une performance économique moins bonne que les grandes (productivité, profit, innovation et améliorations technologiques) et qu'elles offrent de moins bonnes conditions de travail, les auteurs scrutent les monographies nationales pour voir comment les PME peuvent compenser leurs désavantages. Trois options sont retenues.

- le recours à l'intervention gouvernementale: formation professionnelle appropriée aux artisans, exclusivité de certaines productions, subventions directes (pas très haut dans l'appréciation des auteurs), déréglementation. Sur ce dernier thème, des précautions sont à prendre: la dérèglementation peut accentuer les écarts de conditions de travail, augmentant ainsi la difficulté pour les PME à conserver leur main-d'oeuvre qualifiée. Cela peut donc inciter les entrepreneurs des PME à miser uniquement sur leur avantage coût, négligeant l'innovation et l'adaptation. Cela pourrait aussi inciter les grandes entreprises à externaliser leurs risques (sous-traitance...) ce qui, à court terme, favoriserait lemploi dans les PME mais serait incompatible avec le bien-être à long terme.

- le développement de liens avec les grandes entreprises. Cette section essaie de démêler ce qu'il y a de dépendance, de subordination, de domination dans les relations entre grandes et petites entreprises. Des exemples, tant positifs que négatifs sont donnés. Une typologie de la décentralisation des opérations de la grande entreprise est fournie: décentralisation de la production vers des unités plus petites, mais toujours sous même propriété, franchisation de la production (abandon de la propriété, mais garantie de flux de revenus) et finalement, désintégration de la production (sous-traitance).

- le développement de structures communautaires de support. La logique économique et la logique socio-politique sont étudiées. Sous le premier thème, on retrouve l'analyse conventionnelle des économies d'échelle possible, (approvisionnement, mise en marché,...) mais aussi une analyse de la masse critique nécessaire au fonctionnement d'un marché du travail aoccupationnel» (assurant ainsi la mobilité de la main-d'oeuvre nécessaire aux PME). Sous le second thème, les auteurs analysent le pouvoir de pression face aux autorités gouvernementales, mais aussi le pouvoir de discipliner la concurrence entre PME à court terme. Une très rapide analyse des lois anti-cartel, dans les districts industriels, est esquissée.

C'est cette dernière section du chapitre d'introduction qui est la plus riche en pistes de réflexion pour tous ceux que la question du développement industriel et régional intéresse. Étant donné l'engouement actuel pour les "grappes industrielles", cette section présente un cadre d'analyse qui manquait.

Les différentes monographies nationales qui suivent le chapitre d'introduction se lisent avec grand intérêt. Même si chaque chercheur partait avec la même liste de questions, les monographies sont très différentes les unes des autres. Chaque chercheur était en effet dépendant de la littérature existante sur le sujet dans son pays. La lecture de l'ensemble des monographies nous permet donc de saisir la diversité des problématiques entourant les PME et vient donc enrichir la compréhension du phénomène. 